\title{
The Efficient Set Mathematics When Mean-Variance Problems Are Subject to General Linear Constraints
}

\author{
Michael J. Best and Robert R. Grauer *
}

In this paper we develop the efficient set mathematics for the case where mean-variance portfolio problems are subject to general linear constraints. We derive closed-form expressions for the optimal portfolio, the associated multipliers, and the portfolio's expected return and variance in each interval where the active set changes; identify where kinks on the efficient frontier occur; and show that securities plot on a security market hyperplane. The analysis extends our knowledge of portfolio theory, clarifies the zerobeta Capital Asset Pricing Model and the conditions under which securities plot on the Security Market Line (SML), and provides insight into the ambiguities associated with using the SML criterion to measure investment performance.

\section{Introduction}

Markowitz (1956, 1959) and Sharpe (1970) analyzed Mean-Variance (MV) portfolio problems subject to general linear constraints, employing Quadratic Programming (QP) and Parametric Quadratic Programming (PQP) methods. Subsequently, the primary focus of research in finance shifted from normative models of portfolio selection to equilibrium models of asset pricing, in which the only binding constraint is that the portfolio weights sum to one. In this market equilibrium setting Sharpe (1964) and Lintner (1965) showed that securities plot on the Security Market Line (SML), and Merton (1972) developed analytic solutions for the optimal portfolios and the minimum variance frontier. Roll (1977) emphasized the equivalence of the statements that the market portfolio is MV efficient, and securities plot on the SML. Ross (1977) reversed the trend to the single-constraint case. He suggested that without a riskless asset and in the presence of short sales (nonnegativity) constraints there are no "kinks", i.e., points of nondifferentiability, on the MV-efficient frontier. Dybvig (1984) showed that kinks may occur in this case, although his analysis suggested they may be a rarity.

In this paper we return to a PQP framework to develop the efficient set mathematics

* Department of Combinatorics and Optimization, University of Waterloo, and Department of Economics, Faculty of Business Administration, Simon Fraser University.

Address reprint requests to Robert R. Grauer, Department of Economics, Simon Fraser University, Burnaby, British Columbia V5A 1 S6. 
in the case where MV problems are subject to general linear constraints. While the PQP framework is usually associated with some type of computer algorithm, we employ it to develop the analytics of the problem. The framework has several advantages. First. the efficient set mathematics for any MV problem follows directly from the optimality conditions of a PQP problem and the definitions of the expected return and variance of a portfolio. Second, the framework allows us to generalize the traditional efficient set mathematics in a straightforward manner; all previous analyses represent special cases. The efficient set mathematics of Merton and Roll and the SML of Sharpe and Lintner follow as a special case, where the only constraint is that the portfolio weights sum to one. The analysis of kinks by Ross and Dybvig is also a special case, where short sales constraints are added to the constraint that the portfolio weights sum to one. Third, discussions of the analytics of MV portfolio problems, which usually are associated with some form of equilibrium model of asset pricing and contain only one constraint. are almost completely divorced from discussions of the computational aspects of MV portfolio problems where other constraints may well be binding. This unfortunate split is overcome in the PQP formulation. which provides a natural framework for discussing both the analytical and computational aspects of MV portfolio problems.'

Even so, if under most circumstances the budget constraint turned out to be the only binding constraint, and, if constrained portfolio optimization did not enhance portfolio performance, there would be no compelling financial reasons to explore the efficient set mathematics subject to general linear constraints. But recent evidence indicates what users of portfolio optimizers may have always suspected: other constraints will almost certainly be binding, except under very special circumstances. (See Best and Grauer (1988, 1989).) Furthermore, a small but growing body of evidence indicates that it may be possible to earn economically and statistically significant excess returns employing constrained portfolio optimizing techniques. (See, for example, Grauer and Hakansson (1986, 1987, 1990) and Grauer, Hakansson, and Shen (1990).) So, from a portfolio theory point of view. there are clear reasons for wishing to extend the efficient set mathematics. Moreover, if investors choose portfolios in which constraints (other than the budget constraint) are binding, the mathematics will have important implications for, and will provide important insights into, models of asset pricing and measures of investment performance, as we show below.

The paper proceeds as follows. In section II we examine MV problems with no riskless assets. In part A we formulate the MV problem as a $P Q P$ problem, outlining the $\mathrm{PQP}$ problem's most basic properties. For readers unfamiliar with $\mathrm{PQP}$ problems we note that this basic material is much simpler than it may first appear. Investment of a little time here pays large dividends, as the PQP framework permits a simple and direct generalization of the analytics of the well-known single-constraint efficient set mathematics. For expositional purposes we begin with a review of the single-constraint case, employing the PQP framework, in part $\mathrm{B}$. Then we provide a very straightforward exten-

'The PQP framework also easily handles closely related problems. For example, Roll's (1980) orthogonal portfolios and orthogonal frontier, which together constitute the set of minimal variance portfolios orthogonal to (or equivalently uncorrelated with) some portfolio, can be derived easily from a PQP problem subject to two linear constraints. Similarly, Dybvig and Ross's (1985) analysis of performance measurement and the SML relies on a minimal variance frontier of portfolios that are both MV-efficient and plot on an SML defined relative to an inefficient portfolio. Again, these portfolios and the associated frontier can be derived easily from a PQP problem subject to two linear constraints. Finally, the PQP formulation provides a natural framework for the closely related sensitivity analysis of MV portfolio problems. (See footnotes 4 and 14). 
sion to the general-linear-constraints case in part $\mathrm{C}$. We derive closed-form expressions for the optimal portfolio, the associated multipliers, and the portfolio's expected return and variance in each interval in which the active set changes, generalizing the efficient set mathematics of Merton and Roll. ${ }^{2}$ In part D we identify regions where kinks on the efficient frontier occur, generalizing Dybvig's analysis. The section also both expands and clarifies Markowitz's (1959) discussion of kinks. In part E and in section III, which discusses MV problems with either one or two riskless assets, we show that security means plot on a hyperplane spanned by a vector of betas and the gradients of the active constraints. At the market equilibrium level constraints on borrowing and lending, which arise from divergent borrowing and lending rates, give rise to the zero-beta Capital Asset Pricing Model (CAPM) of Black (1972) and Brennan (1971). Our analysis clarifies the zero-beta model and the conditions under which securities plot on the SML, and provides insights into the ambiguities associated with using the SML criterion to measure investment performance. We summarize the paper in section IV.

\section{MV Problems With No Riskless Assets}

\section{A. Formulation of the Problem}

Consider the Markowitz model with no riskless asset, subject to general linear constraints,

$$
\max \left\{t \boldsymbol{\mu}^{\prime} \boldsymbol{x}-\boldsymbol{x}^{\prime} \boldsymbol{\Sigma} \boldsymbol{x} / 2 \mid \boldsymbol{A x} \leq \boldsymbol{b}\right\},
$$

where $\boldsymbol{\mu}$ and $\boldsymbol{x}$ are $n$-vectors composed of one plus expected rates of return and portfolio weights, respectively, $\Sigma$ is an $(n, n)$-positive definite covariance matrix (i.e., we assume away degenerate cases where $\Sigma$ is not of full rank as they might permit arbitrage), $\boldsymbol{A}$ is an $(m, n)$ constraint matrix, $b$ is an $m$-vector, and throughout any vector $y$ is assumed to be a column vector unless indicated to the contrary by transposition (e.g., $\left.y^{\prime}\right){ }^{3,4}$ There are a number of ways to interpret equation (1). It may be interpreted as a PQP problem in which the minimum variance frontier is traced out as the PQP parameter $t$ varies from $-\infty$ to $\infty .^{5}$ However, positive values of $t$ are of primary economic

${ }^{2}$ Ex ante we do not know which constraints will be active in an interval, hence the need for a PQP algorithm. But knowing which constraints are active in an interval allows us to develop closed-form expressions for the solution, the associated multipliers, and the portfolio's expected return and variance, and to better understand the properties of the system.

${ }^{3}$ We do not give explicit consideration to equality constraints in equation (1). This is for simplicity of notation and presentation. Equality constraints can easily be accounted for by observing that their indices are always in the active set and their associated multipliers are not restricted in sign. See Best and Ritter (1985).

${ }^{4} \mathrm{~A}$ more general PQP problem subject to general linear constraints may be written as

$$
\max \left\{-(\boldsymbol{c}+t \boldsymbol{q})^{\prime} \boldsymbol{x}-\boldsymbol{x}^{\prime} \boldsymbol{C} \boldsymbol{x} / 2 \mid \boldsymbol{A x} \leq \boldsymbol{b}+\boldsymbol{t} \boldsymbol{p}\right\}
$$

where $c$ is an $n$-vector, $C$ is an $(n, n)$-matrix, and $p$ an $m$-vector. See Best (1982). Equation (1) is a special case of the more general $\mathrm{PQP}$ with $\boldsymbol{c}=\mathbf{0}, \boldsymbol{q}=-\boldsymbol{\mu}, \boldsymbol{C}=\boldsymbol{\Sigma}$, and $\boldsymbol{p}=\mathbf{0}$. The more general $\mathrm{PQP}$ problem allows an investor with a fixed risk-tolerance parameter to perform sensitivity analysis for $\mathrm{MV}$ portfolio problems in which either the mean vector or right-hand side of the constraints change. See Best and Grauer (1984, 1985).

${ }^{5}$ Merton (1972) and Roll (1977) developed the efficient set mathematics for the single constraint case by minimizing the variance for all levels of expected return. It is easily verified from the optimality conditions that the parameter $t$ from equation (1) is equivalent to the multiplier associated with the constraint $\mu_{p}=\mu^{\prime} \boldsymbol{x}$ in their formulation. 
interest as they yield MV efficient portfolios. In this case it is convenient to think of $t$ as an MV investor's risk tolerance parameter, where the larger $t$ the more tolerant the investor is to risk. ${ }^{6}$ Equation (1) is also open to an interesting interpretation that links it to von Neumann-Morgenstern utility and the isoelastic or power utility functions $u(w)=w^{\gamma} / \gamma, \gamma<1$. Using a Taylor series approximation to expected utility for short holding periods, we can show that $t$ is equal to the reciprocal of the Pratt-Arrow measure of relative risk aversion (RRA): hence, the terminology risk tolerance parameter. (See, e.g., Ohlson (1975), Pulley (1981), and Grauer (1986).) That is, $t=1 / R R A$, where $R R A=-w u^{\prime \prime}(w) / u^{\prime}(w)$ is evaluated at a zero rate of return. For the isoelastic or power functions, $R R A=1-\gamma$, and the $\mathrm{MV}$ approximations to them are given with $t=1 /(1-\gamma)$. Under certain conditions this result holds exactly in continuous time (see, e.g., Merton (1980)). Finally, we note that if $t$ is fixed at some value, say, $t=T$. then equation (1) is a QP problem where an investor with risk tolerance parameter $T$ maximizes his or her MV utility subject to the constraints. The investor's indifference curves are linear in MV space and have slope $1 /(2 T)$.

Although a PQP algorithm is required to solve almost any type of realistic $\mathrm{MV}$ investment problem, we require only the most basic properties of $P Q P$ problems to develop the analysis. ${ }^{7}$ Associated with the solution of a PQP is a set of intervals $t_{0} \leq t \leq t_{1}, t_{1} \leq t \leq t_{2}, \ldots, t_{v-1} \leq t \leq t_{v}$. Each interval corresponds to a different set of active (or binding) constraints. At the end of a typical interval, a previously inactive constraint becomes active, or a previously active constraint becomes inactive. ${ }^{8}$ In the $i$ th interval, the optimal solution $\boldsymbol{x}_{i}(t)$ and associated multipliers $\lambda_{i}(t)$ are linear functions of the parameter $t$. Thus there are vectors $h_{0 i}, h_{1 i}, g_{0 i}$ and $g_{1 i}$ such that

$$
\boldsymbol{x}_{i}(t)=\boldsymbol{h}_{0 i}+t h_{1 i}, \quad \text { and } \quad \lambda_{i}(t)=g_{0 i}+t g_{1 i},
$$

for all $t$ with $t_{i} \leq t \leq t_{i+1} .{ }^{9}$ Each $t_{i}$ corresponds to a "corner" portfolio. The vector $h_{0 i}$ is the nonvarying portion of the solution and $t h_{1 i}$ is the varying portion of the solution. In all portfolio problems the budget constraint dictates that the portfolio weights sum to one, i.e., $\iota \boldsymbol{\iota}^{\prime} \boldsymbol{x}_{i}=1$, where $\iota$ is a vector of ones. This implies that the nonvarying portion of the portfolio must sum to one and the varying portion of the portfolio must sum to zero, i.e., $\iota^{\prime} \boldsymbol{h}_{0 i}=1$ and $\iota^{\prime} \boldsymbol{h}_{1 i}=0$.

In a typical parametric interval, where $t_{i} \leq t \leq t_{i+1}$, the active constraints hold as equalities, i.e., $A_{i} \boldsymbol{x}=\boldsymbol{b}_{i}$, where, if $k$ denotes the number of constraints which are active in the interval, $\boldsymbol{A}_{i}$ is a $(k, n)$ matrix, whose rows are $k$ of the rows of $\boldsymbol{A}$, and $\boldsymbol{b}_{\boldsymbol{i}}$ is a $k$-vector, whose components are the corresponding $k$ components of $\boldsymbol{b}$. The optimal solution, $\boldsymbol{x}_{i}(t)$, and associated multipliers, $\lambda_{i}(t)$, for equation (1) are the solutions of

"In this MV framework the terms "utility function," "risk tolerance," and "risk aversion" are not to be confused with von Neumann-Morgenstern utility nor with the Pratt-Arrow measures of risk aversion. (See Sharpe 1970,1985 .) On the other hand, the MV utility function is consistent with the expected utility theorem in a single period if the investor has negative exponential utility and makes normal probability assessments.

${ }^{7}$ This paper is not concerned with solution procedures for QP or PQP algorithms. While the critical line algorithm of Markowitz $(1956,1959)$ is probably the best-known algorithm in the finance literature, Best (1984) shows the equivalence of several QP algorithms.

${ }^{8}$ A complete analysis must account for some other possibilities: such as exchanging two constraints, or determining that equation (1) is unbounded from above or infeasible for $t>t_{i+1}$. We refer the reader to Best (1982) for a detailed analysis of these additional cases.

${ }^{9}$ Because equation (1) has less-than-or-equal-to constraints, the $k$-dimensional vector of multipliers is nonnegative, i.e., $\boldsymbol{\lambda}_{i}(t) \geq \mathbf{0}$ for $t_{i} \leq t \leq t_{i+1}$. For completeness we note that, if $k<m$, the $m-k$ inactive constraints have zero multipliers. 
the linear equations

$$
\left[\begin{array}{cc}
\Sigma & A_{i}^{\prime} \\
A_{i} & 0
\end{array}\right]\left[\begin{array}{l}
x_{i}(t) \\
\lambda_{i}(t)
\end{array}\right]=\left[\begin{array}{l}
0 \\
b_{i}
\end{array}\right]+t\left[\begin{array}{l}
\mu \\
0
\end{array}\right]
$$

The efficient set mathematics for MV problems follow as special cases of the PQP problem (1), the Karush-Kuhn-Tucker or optimality conditions (3), and the definitions of the expected return and variance of a portfolio. ${ }^{10,11}$

\section{B. The Single-Constraint Case}

The simplest MV problem, for which the only constraint is the budget constraint, has been the primary focus of analysis. (See Black (1972), Lintner (1965), Merton (1972), Roll (1977), and Sharpe (1964).) For expositional purposes we review the single-constraint case, employing a PQP framework in this section, and then provide a very straightforward extension to the general-linear-constraints case in part $C$. In the single constraint case the optimal solution, the multiplier, and the expected return and variance of the optimal portfolio should all carry zero subscripts to be consistent with the notation employed in the general-linear-constraints case. But to keep the notation as simple as possible we suppress the zero subscripts. The PQP problem is a special case of equation (1) with $\boldsymbol{A}=\boldsymbol{\iota}^{\prime}$ and $\boldsymbol{b}=1$, namely

$$
\max \left\{\boldsymbol{t}^{\prime} \boldsymbol{x}-\boldsymbol{x}^{\prime} \mathbf{\Sigma} \boldsymbol{x} / 2 \mid \iota^{\prime} \boldsymbol{x}=1\right\}
$$

In this special case the optimality conditions (3) become

$$
\Sigma x+\imath \lambda=t \mu, \quad \iota x=1
$$

Solving these relations gives the optimal solution and associated multiplier:

$$
\begin{aligned}
& x(t)=\Sigma^{-1} \iota / c+t\left[\Sigma^{-1}(\mu-\iota a / c)\right], \\
& \lambda(t)=-1 / c+t a / c .
\end{aligned}
$$

By definition the efficient set constants $a, b$, and $c$ are

$$
a=\iota^{\prime} \Sigma^{-1} \mu, \quad b=\mu^{\prime} \Sigma^{-1} \mu, \quad \text { and } c=\iota^{\prime} \Sigma^{-1} \iota .
$$

From equations (2), (6), and (7) it is obvious that $x(t)$ and $\lambda(t)$ are linear in $t$. Also, $h_{0}=$ $\Sigma^{-1} \iota / c$ is the nonvarying portion of the optimal portfolio- the global minimum variance portfolio in this case- whose components sum to one. The vector $t h_{1}=t\left[\Sigma^{-1}(\mu-\right.$ $\iota a / c)]$ is the varying portion of the optimal portfolio, whose components sum to zero.

${ }^{10}$ The Karush-Kuhn-Tucker conditions (3) are both necessary and sufficient for optimality. See Mangaserian (1969).

"I Our closed-form expressions provide a major extension of the efficient set mathematics. But given their PQP formulation of the problem much of what we (or anyone else) have to say about MV problems was implicit in Markowitz (1956, 1959) and Sharpe (1970). 
The expected return, $\mu_{p}=\boldsymbol{\mu}^{\prime} \boldsymbol{x}$, and variance, $\sigma_{p}^{2}=\boldsymbol{x}^{\prime} \boldsymbol{\Sigma} \boldsymbol{x}$, of the optimal portfolio are linear and quadratic functions of $t$ :

$$
\mu_{p}=\alpha_{0}+\alpha_{1} t, \quad \text { and } \quad \sigma_{p}^{2}=\gamma_{0}+\gamma_{1} t+\gamma_{2} t^{2} \text {, }
$$

respectively, where

$$
\begin{aligned}
& \alpha_{0}=\boldsymbol{\mu}^{\prime} \boldsymbol{h}_{0}=a / c, \quad \alpha_{1}=\boldsymbol{\mu}^{\prime} \boldsymbol{h}_{1}=b-a^{2} / c, \\
& \gamma_{0}=\boldsymbol{h}_{0}^{\prime} \boldsymbol{\Sigma} \boldsymbol{h}_{0}=1 / c, \quad \gamma_{1}=2 \boldsymbol{h}_{0}^{\prime} \boldsymbol{\Sigma} \boldsymbol{h}_{1}=0, \quad \text { and } \quad \gamma_{2}=\boldsymbol{h}_{1}^{\prime} \boldsymbol{\Sigma} \boldsymbol{h}_{1}=\alpha_{1} .
\end{aligned}
$$

We note that $\alpha_{0}$ is the mean of the nonvarying portion of the portfolio, and $\alpha_{1} t$ is the mean of the varying portion of the portfolio. Likewise, $\gamma_{0}$ is the variance of the nonvarying portion of the portfolio, $\gamma_{1} t$ is twice the covariance between the nonvarying and varying portions of the portfolio, and $\gamma_{2} t^{2}$ is the variance of the varying portion of the portfolio. The expected return on the portfolio increases as the optimal portfolio changes from the minimum variance portfolio, and, because $\gamma_{1}=0$, the variance of the optimal portfolio is simply the variance of the nonvarying portion of the portfolio plus the variance of the varying portion of the portfolio.

Eliminating $t$ from equation (8) we obtain the equation of the minimum variance frontier

$$
\left(\mu_{p}-\alpha_{0}\right)^{2}=\alpha_{1}\left(\sigma_{p}^{2}-\gamma_{0}\right),
$$

which is a parabola (hyperbola) in MV (mean-standard deviation) space. For $t \geq 0$, equation (9) is the equation of the efficient frontier. From equation (8) and the Chain Rule of Calculus the slope of the efficient frontier is $d \mu_{p} / d \sigma_{p}^{2}=1 /(2 t)$, provided $t \neq 0$. SML

Rearranging the first partition of equation (5) we obtain two equivalent forms of the

$$
\mu=\lambda / t \mathbf{t}+1 / t \Sigma x
$$

and

$$
\mu=r_{z} \iota+\left(\mu_{p}-r_{z}\right) \beta,
$$

where $r_{z}=\lambda / t, \beta=(\Sigma x) / \sigma_{p}^{2}$, and the $j$ th element of $\boldsymbol{\Sigma} x$ is the covariance of the return on security $j$ with the return on the optimal portfolio.

\section{The General-Linear-Constraints Case}

With general linear constraints the PQP problem is given by equation (1). In the $i$ th interval, where $t_{i} \leq t \leq t_{i+1}$, the optimality conditions include equation (3). The solution and associated multipliers are

$$
\boldsymbol{x}_{i}(t)=\Sigma^{-1} A_{i}^{\prime} c_{i}^{*-1} b_{i}+t\left[\Sigma^{-1}\left(\mu-A_{i}^{\prime} c_{i}^{*-1} a_{i}^{*}\right)\right]
$$




$$
\lambda_{i}(t)=-c_{i}^{*-1} b_{i}+t c_{i}^{*-1} a_{i}^{*}
$$

where we define

$$
a_{i}^{*}=A_{i} \Sigma^{-1} \mu, \text { and } c_{i}^{*}=A_{i} \Sigma^{-1} A_{i}^{\prime} \text {. }
$$

Clearly, $a_{i}^{*}$, a $k$-vector, and $c_{i}^{*}$, a $(k, k)$-matrix, ${ }^{12}$ are the analogs of the efficient set (scalar) constants $a$ and $c$. Similarly $\boldsymbol{A}_{i}$, a $(k, n)$-matrix of constraint coefficients, replaces $\iota^{\prime}$, the $n$-vector of ones from the budget constraint, and the right-hand side vector $b_{i}$ replaces the right-hand side (the scalar one) of the budget constraint. By analogy to the single constraint case, $h_{0 i}$ is the global minimum-variance portfolio subject to the binding constraints in the $i$ th interval, and, because the budget constraint is one of the $k$ active constraints, $\iota ' h_{0 i}=1$, and $\iota \iota^{\prime} h_{l i}=0$.

The expected return, $\mu_{p i}=\boldsymbol{\mu}^{\prime} \boldsymbol{x}_{i}$, and variance, $\sigma_{p i}^{2}=\boldsymbol{x}_{i}^{\prime} \boldsymbol{\Sigma} \boldsymbol{x}_{i}$, of portfolio $p$ are linear and quadratic functions of $t$ :

$$
\mu_{p i}=\alpha_{0 i}+\alpha_{1 i} t, \text { and } \sigma_{p i}^{2}=\gamma_{0 i}+\gamma_{1 i} t+\gamma_{2 i} t^{2}
$$

respectively, where upon simplification,

$$
\begin{aligned}
& \alpha_{0 i}=\boldsymbol{\mu}^{\prime} \boldsymbol{h}_{0 i}=\boldsymbol{a}_{i}^{* \prime} \boldsymbol{c}_{i}^{*-1} \boldsymbol{b}_{i}, \quad \alpha_{1 i}=\boldsymbol{\mu}^{\prime} \boldsymbol{h}_{1 i}=b-\boldsymbol{a}_{i}^{* \prime} \boldsymbol{c}_{i}^{*-1} \boldsymbol{a}_{i}^{*}, \\
& \gamma_{0 i}=\boldsymbol{h}_{0 i}^{\prime} \Sigma \boldsymbol{h}_{0 i}=\boldsymbol{b}_{i}^{\prime} \boldsymbol{c}_{i}^{*-1} \boldsymbol{b}_{i}, \quad \gamma_{1 i}=2 \boldsymbol{h}_{0 i}^{\prime} \boldsymbol{\Sigma}_{1 i}=0, \quad \gamma_{2 i}=\boldsymbol{h}_{1 i}^{\prime} \Sigma \boldsymbol{h}_{1 i}=\alpha_{1 i},
\end{aligned}
$$

or

$$
\mu_{p i}=\alpha_{0 i}+\alpha_{1 i} t \text {, and } \sigma_{p i}^{2}=\gamma_{0 i}+\alpha_{1 i} t^{2}
$$

The expressions for the $\alpha$ 's and $\gamma$ 's in equations (14) and (15) are obvious generalizations of the single-constraint case in equation (8). Again $a_{i}^{*}$ and $c_{i}^{*}$ replace the efficient set scalar constants $a$ and $c$, and the right-hand side vector $b_{i}$ replaces the right-hand side (the scalar one) of the budget constraint. Eliminating $t$ from equation (15) yields

$$
\left(\mu_{p i}-\alpha_{0 i}\right)^{2}=\alpha_{1 i}\left(\sigma_{p i}^{2}-\gamma_{0 i}\right) \text {. }
$$

We call equation (16) the $i$ th interval frontier. The points from equation (16), where $t_{i} \leq t \leq t_{i+1}$, are part of the efficient frontier. The efficient frontier itself is a piecewise parabola in MV space composed of the relevant $\mu_{p i}, \sigma_{p i}^{2}$ pairs in each interval. From the Chain Rule of Calculus and equation (15), the slope of an interval frontier is $d \mu_{p i} / d \sigma_{p i}^{2}=\alpha_{1 i} /\left(2 \alpha_{1 i} t\right)=1 /(2 t)$, provided $\alpha_{1 i} \neq 0$. For $t>0$ and $\alpha_{1 i} \neq 0$, the slope is positive and decreases as $t$ increases. In this case, adjacent interval frontiers are tangent at the point corresponding to their common corner portfolio. Therefore, the

${ }^{12}$ We assume that there are no redundant constraints, i.e., $A_{i}$ is of rank $k$. Therefore, $\boldsymbol{A}_{i} \Sigma^{-1} \boldsymbol{A}_{i}^{\prime}$ is a symmetric-positive-definite matrix. 


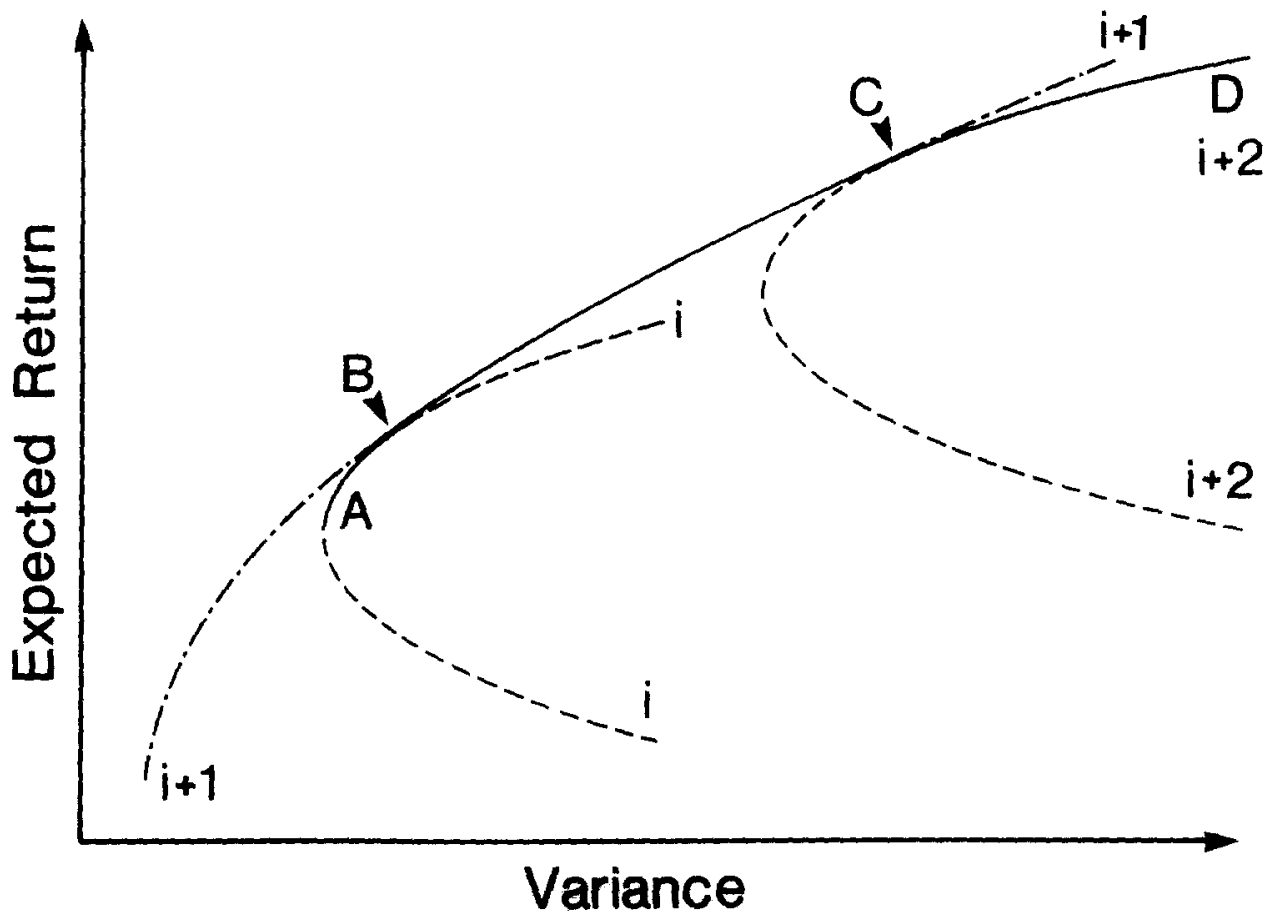

Figure 1. A Mean-Variance Efficient Frontier subject to general linear constraints. Interval frontiers are labeled $i, i+1$, and $i+2$, and the efficient frontier is labeled $A B C D$. A multiplier is reduced to zero at $B$ and a constraint becomes active at $C$.

efficient frontier is continuously differentiable with slope $1 /(2 t)$. Figure 1 illustrates this case with three interval frontiers labeled $i, i+1$, and $i+2$, and the efficient frontier labeled $A B C D$. Changes in the active set occur when a multiplier is reduced to zero at point $B$, i.e., an active constraint becomes inactive, and an inactive constraint becomes active at $\mathrm{C}$.

\section{Kinks on the Efficient Frontier}

Kinks, i.e., points of nondifferentiability, on the efficient frontier occur under the following conditions.

\section{Theorem}

Given $t_{i+1}>t_{i}$, the following are equivalent:

(i) the efficient frontier has a kink;

(ii) $x_{i}(t)$ is constant, i.e., $h_{1 i}=0$, for $t_{i} \leq t \leq t_{i+1}$;

(iii) $\mu=A_{i}^{\prime} g_{1 i}$.

Proof: The slope of an interval frontier is $d \mu_{p i} / d \sigma_{p i}^{2}=\alpha_{1 i} /\left(2 \alpha_{1 i} t\right)=1 /(2 t)$, provided $\alpha_{1 i} \neq 0$ and $t \neq 0$. Kinks occur when $\alpha_{1 i}=0$. From equation (14), $\gamma_{2 i}=\alpha_{1 i}$. Then, because $\gamma_{2 i}=\boldsymbol{h}_{1 i}^{\prime} \Sigma \boldsymbol{h}_{1 i}$ and $\Sigma$ is positive definite, $\alpha_{1 i}=0$ is equivalent to $\boldsymbol{h}_{1 i}=\mathbf{0}$. This in turn is equivalent to $x_{i}(t)$ being constant throughout the interval $t_{i} \leq t \leq t_{i+1}$, with $t_{i+1}>t_{i}$. Purthermore, the first partition of equation (3) together with equation (2) 
implies

$$
\Sigma \boldsymbol{h}_{1 i}+\boldsymbol{A}_{i}^{\prime} \boldsymbol{g}_{1 i}=\boldsymbol{\mu}, \text { and } \boldsymbol{\Sigma} \boldsymbol{h}_{0 i}+\boldsymbol{A}_{i}^{\prime} \boldsymbol{g}_{0 i}=\mathbf{0}
$$

Thus, $h_{1 i}=\mathbf{0}$ if and only if $\mu=A_{i}^{\prime} g_{1 i}$. That is, the composition of the optimal portfolio is constant in an interval if and only if the mean vector is in the space spanned by the gradients of the active constraints. When $\boldsymbol{h}_{1 i}=\mathbf{0}$ it is also apparent that $\boldsymbol{\Sigma} \boldsymbol{x}_{i}=\boldsymbol{\Sigma} \boldsymbol{h}_{0 i}=$ $-\boldsymbol{A}_{i}^{\prime} \boldsymbol{g}_{0 i}$.

Figure 2 illustrates a kink. The efficient frontier is labelled ABC. The curve $i$ is efficient until a constraint becomes active when $t=t_{i+1}$ at the point $\mathrm{B}$. In the interval $i+1$ the efficient portfolio remains constant and the interval frontier collapses to a single point, namely the point $B$, on the efficient frontier. At this point, the left-hand derivative $\left(2 t_{i+1}\right)^{-1}$ is strictly greater than the right-hand derivative $\left(2 t_{i+2}\right)^{-1}$. Thus the efficient frontier is not differentiable at this point, or equivalently a kink exists. At $t=t_{i+2}$ some positive component of $\lambda_{i+1}(t)$ is reduced to zero and $\mu$ no longer lies in the span of $A_{i+1}^{\prime} g_{1 i+1}{ }^{13}$ Then in the interval $i+2$, where $t>t_{i+2}$, the associated constraint is inactive, and $x_{i+2}(t)$ again varies with $t .{ }^{14}$

Dybvig (1985) succinctly characterized kinks for the no-riskless-asset and no-shortsales case: "the mean-variance frontier can have a kink but only if the included securities all have the same expected return." The result follows easily from our theorem. The parametric formulation of the model considered by Dybvig is

$$
\max \left\{t_{\mu}^{\prime} x-\left(x^{\prime} \boldsymbol{\Sigma} x\right) / 2 \mid \iota^{\prime} x=1, x \geq 0\right\}
$$

In any parametric interval, the budget constraint together with some nonnegativity constraints will be active. Thus the columns of $\boldsymbol{A}_{i}^{\prime}$ will include $\iota$ together with unit vectors (negated) corresponding to the active nonnegativity constraints. By convention assume the first constraint is $\iota^{\prime} \boldsymbol{x}=1$, and for simplicity of notation let the components of $\boldsymbol{g}_{1 i}$ be $v_{j}, j=0, \ldots, k-1$. Parts (i) and (iii) of the above theorem together assert that a kink occurs if and only if

$$
\mu_{j}=v_{0} \text {, for each security } j \text { held positively, }
$$

and

$\mu_{j}=v_{0}-v_{j}$, for each security $j$ held at level zero.

${ }^{13}$ Ross (1977, p. 180) suggested, "Equation (10) also disproves a common fallacy in drawing the efficient set for risky assets with no short sales. At a switching point where the portfolio composition changes and a new asset, say, $i$ enters while $j$ leaves, $\delta_{i}=0$ and $\delta_{j}=0$; since $\zeta$ is the slope on the frontier, there is no 'kink'." ( $\delta_{i}$ and $\delta_{j}$ are multipliers and $\zeta$ corresponds to our parameter $t$ ). Our analysis shows that the two multipliers are not equal to zero simultaneously. It is precisely this case, in which the portfolio is constant in an interval and a multiplier is being reduced to zero, that produces kinks on the efficient frontier.

${ }^{14}$ After $t$ increases past a certain value in each constrained MV problem the solution does not change. For example, with budget and nonnegativity constraints only, after $t$ reaches a certain value everything is invested in the highest return asset. In this final interval the efficient frontier is a point where the slope is undefined. The same phenomenon occurs with sensitivity analysis on the means except that this last interval traces out a vertical line on an investor's Mean-Parameterized Portfolio Expansion Path instead of a point on the MV-efficient frontier. Furthermore, while the investor's Portfolio Expansion Path is piecewise parabolic, it may contain negatively sloping segments and is characterized by types of kinks not found in traditional MV analysis. See Best and Grauer $(1984,1985)$. 


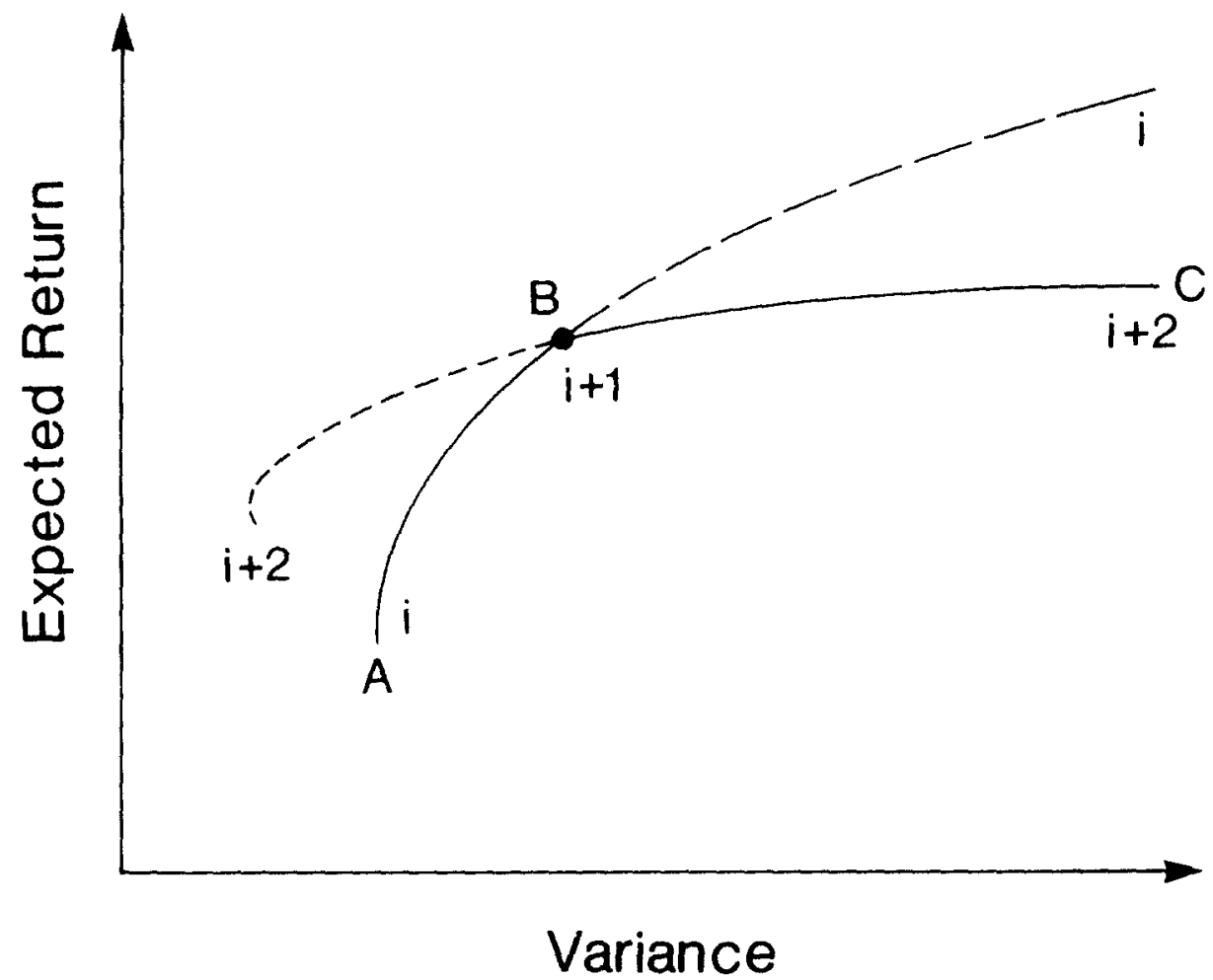

Figure 2. A Mean-Variance Efficient Frontier with a kink. Interval frontiers are labeled i, $i+1$, and $\mathrm{i}+2$, and the efficient frontier is labeled $A B C$. The kink occurs in the interval $i+1$ (the point B) where the composition of the optimal portfolio does not vary.

The first condition states that all positively held securities must have the same expected return, namely $v_{0}$.

Dybvig's results may leave the impression that kinks are rare and occur only when a subset of securities has equal means. In general, the impression is false. The existence of kinks depends heavily on the constraints of the problem. The more constraints there are, the greater the probability of a kink. Kinks may occur at an extreme point, where $n$ constraints are active, ${ }^{15}$ or at a point where $k<n$ constraints are active. Consider the case in which an efficient portfolio is an extreme point. Then $A_{i}$ is square and nonsingular so that part (iii) of the theorem is satisfied with $g_{1 i}=\left(A_{i}^{-1}\right)^{\prime} \mu$, and there is a kink at the corresponding point on the efficient frontier. Depending on the specific form of the constraints, as few as one and as many as $n$ securities may be held, even though all $n$ expected returns differ. Now consider the case with $k<n$ active constraints. Here it may appear that the generalization of Dybvig's result is that the $n-k$ securities not at their bounds must have equal expected returns. This is correct if the budget constraint and simple upper and lower bounds on individual securities are the only constraints. But in general the condition that $\boldsymbol{\mu}=\boldsymbol{A}_{i}^{\prime} \boldsymbol{g}_{1 i}$, with $k<n$ constraints active, does not preclude a kink where all $n$ expected returns differ and all $n$ securities are held. ${ }^{15}$ More precisely, there must be at least $n$ active constraints that have linearly independent gradients. See
Best and Ritter (1985). 


\section{E. The Security Market Hyperplane}

In this section we simply rearrange the first partition of the optimality conditions (3) to show that in general securities plot on a security market hyperplane. The results in this and the next section clarify the zero-beta CAPM and the conditions under which securities plot on the SML, and provide insights into ambiguities associated with using the SML criterion of investment performance. For notational simplicity we drop the subscript $i$ from the components of $A_{i}, \lambda_{i}$ and $b_{i}$. Let $a_{j}$ be the gradient of the $j$ th active constraint ( $a_{j}^{\prime}$ is the $j$ th row of $A_{i}$ ), $b_{j}$ be the $j$ th component of the right-hand side constraint vector $b_{i}$, and $\lambda_{j}$ be the $j$ th component of the vector of multipliers $\lambda_{i}$. We continue with the convention that the budget constraint $\iota^{\prime} x=1$ is the first constraint, but we number the active constraints from one to $k$. That is, we let $a_{1}=\imath$ and the first element of $b_{i}$ equal 1 . Rearranging the first set of equations (3) we find that

$$
\boldsymbol{\mu}=\boldsymbol{\lambda}_{1} / t \boldsymbol{t}+1 / t \boldsymbol{\Sigma} \boldsymbol{x}+\sum_{j=2}^{k} \boldsymbol{\lambda}_{j} / t \boldsymbol{a}_{j}
$$

or with further minor rearrangement that

$$
\boldsymbol{\mu}=\lambda_{1} / \boldsymbol{t} \boldsymbol{t}+\left(\mu_{p}-\sum_{j=1}^{k} \lambda_{j} / t b_{j}\right) \boldsymbol{\beta}+\sum_{j=2}^{k} \lambda_{j} / t \boldsymbol{a}_{j} .
$$

These equations show that when the MV problem is subject to general linear constraints the underlying security means plot on a hyperplane spanned by either $\boldsymbol{\Sigma x}$ or $\boldsymbol{\beta}$, and also by the gradients of the active constraints weighted by their respective multipliers divided by the risk tolerance parameter $t$. All securities and portfolios plot on this hyperplane. When we project the points on the hyperplane onto mean-beta space, the securities, for which $\iota^{\prime} x=1$ is the only binding constraint, plot on the line

$$
\boldsymbol{\mu}=\lambda_{1} / t+\left(\boldsymbol{\mu}_{p}-\sum_{j=1}^{k} \lambda_{j} / t b_{j}\right) \beta .
$$

The securities whose weights in the optimal portfolio are constrained in any other way plot off the line. In general, the optimal portfolio itself does not plot on the line, and the line is not the SML. ${ }^{16}$ The deviations from the line given by equation (19) are functions of the gradients of the active constraints, the multipliers, and the risk tolerance parameter. For example, suppose the $j$ th active constraint is a simple upper (lower) bound constraint on asset $h$. Then $a_{j}$ is a vector of zeros except $a_{j h}=1(-1)$, and asset $h$ 's mean plots above (below) the line by the distance $\lambda_{j} / t$. It is also clear from equations (17-19) that $\lambda_{1} / t=r_{z}$ is the expected return on a zero-beta portfolio.

In the special case where $\iota x=1$ is the only binding constraint, equation (18) collapses to the SML defined by equation (12). That is, securities plot on the SML if and only if the optimal portfolio is MV efficient subject to the single binding constraint

${ }^{16}$ In the special case where $b_{j}=0, j=2, \ldots, k$, the optimal portfolio does plot on the line. The Black-Brennan zero-beta CAPMs discussed in section III fall in this category. 
that the weights sum to one. Equivalently, securities plot on the SML when the optimality conditions show that the mean vector is spanned solely by a vector of betas (covariances) and a vector of ones, that corresponds to the gradient of the budget constraint. While we focus on security market hyperplanes arising from constrained MV problems here and in the next section, it is also clear that securities do not plot on an SML when taxes or transaction costs alter either the mean vector or the vector of covariances so that the mean vector is no longer spanned solely by vectors of betas (covariances) and ones. The SML hyperplane arising from differential taxes on dividends and capital gains may be the best-known example of this type. (See Brennan (1973) and Litzenberger and Ramaswamy (1979).) Other instances of MV models in which securities do not plot on the traditional SML include models with barriers to international investment (see Stulz 1981), models with transactions costs (see Milne and Smith 1980), and models with infinite transactions costs on human capital (see Mayers 1972).

\section{MV Problems With One Or More Riskless Assets}

With one riskless asset and the budget constraint the relevant PQP problem is

$$
\max \left\{t\left(\mu^{\prime}, r\right)\left[\begin{array}{l}
x \\
x_{r}
\end{array}\right]-\frac{1}{2}\left(\boldsymbol{x}^{\prime}, x_{r}\right)\left[\begin{array}{cc}
\Sigma & 0 \\
\mathbf{0}^{\prime} & 0
\end{array}\right]\left[\begin{array}{l}
\boldsymbol{x} \\
x_{r}
\end{array}\right] \mid\left(\iota^{\prime}, 1\right)\left[\begin{array}{c}
\boldsymbol{x} \\
x_{r}
\end{array}\right]=1\right\},
$$

or alternatively

$$
\max \left\{\boldsymbol{t \mu}^{\prime} \boldsymbol{x}+\operatorname{tr} x_{r}-\boldsymbol{x}^{\prime} \boldsymbol{\Sigma} \boldsymbol{x} /\left.2\right|_{\llcorner} ^{\prime} \boldsymbol{x}+x_{r}=1\right\}
$$

where $x_{r}$ is the fraction of wealth invested in the riskless asset that yields one plus the rate of return $r$. The optimality conditions, the optimal solution, and associated multiplier are

$$
\begin{aligned}
& \Sigma x+\iota \lambda=t \mu, \quad \lambda=t r, \quad \iota^{\prime} x+x_{r}=1, \\
& {\left[\begin{array}{l}
x \\
x_{r}
\end{array}\right]=\left[\begin{array}{l}
0 \\
1
\end{array}\right]+t\left[\begin{array}{c}
\Sigma^{-1}(\mu-r) \\
r c-a
\end{array}\right],} \\
& \lambda=t r .
\end{aligned}
$$

When $t=1 /(a-r c)$ in a market equilibrium setting, equation (22) gives the weights in the market portfolio. The expected return and variance of the optimal portfolios are

$$
\mu_{p}=\alpha_{0}+\alpha_{1} t, \quad \text { and } \quad \sigma_{p}^{2}=\gamma_{2} t^{2}
$$

where

$$
\alpha_{0}=r, \quad \alpha_{1}=b-2 a r+c r^{2}=\gamma_{2} .
$$


The equation of the efficient frontier is the well-known Capital Market Line

$$
\mu_{p}=r+\left(b-2 a r+c r^{2}\right)^{1 / 2} \sigma_{p}
$$

Finally, rearranging the first partition of equation (21) we obtain the SML, where $r$ replaces $r_{z}$ in equation (11).

Now suppose there is risk-free lending at $r_{\ell}$, and risk-free borrowing at $r_{b}$, and we reach the classic market equilibrium first examined by Brennan (1971). In this case the market is MV efficient, as shown in the left-hand panel of Figure 3. But there are three binding constraints at the optimum:

$$
\iota^{\prime} x+x_{\ell}+x_{b}=1, \quad x_{\ell} \geq 0, \quad \text { and } \quad x_{b} \leq 0
$$

where $x_{\ell}$ and $x_{b}$ are the fractions of wealth lent and borrowed, respectively. The optimality conditions include

$$
\left[\begin{array}{l}
\mu \\
r_{\ell} \\
r_{b}
\end{array}\right]=r_{z}\left[\begin{array}{l}
\iota \\
1 \\
1
\end{array}\right]+\mu_{p}-r_{z}\left[\begin{array}{l}
\beta \\
0 \\
0
\end{array}\right]+\lambda_{\ell} / t\left[\begin{array}{c}
0 \\
-1 \\
0
\end{array}\right]+\lambda_{b} / t\left[\begin{array}{l}
0 \\
0 \\
1
\end{array}\right] .
$$

(Black's model is a special case of Brennan's with no riskless borrowing and two binding constraints.) As in previous cases, discussed in section II.E., everything plots on a hyperplane. In mean-beta space, securities whose weights in the optimal portfolio are not constrained (risky securities only in this case) plot on the line labelled $r_{z}$ LmBD in the right-hand panel of Figure 3. Likewise, portfolios containing only risky securities plot on the line. Feasible (infeasible) portfolios that contain securities whose weights in the optimal portfolio are constrained, i.e., portfolios that either borrow or lend, plot below (above) the line; $r_{b}\left(r_{\ell}\right)$ itself plots above (below) the line by the distance $\lambda_{b} / t\left(\lambda_{l} / t\right)$. This line has been identified as the zero-beta SML. The terminology is unfortunate. The line is simply the projection, from equation (26) into mean-beta space, of the securities whose weights in the optimal portfolio are unconstrained. Furthermore, according to the SML criterion for measuring investment performance, MV-efficient portfolios that employ either lending or borrowing (i.e., the portfolios that plot on the line $r_{\ell} L$ or BC) would be branded losers. Yet, these leveraged portfolios MV dominate portfolios of risky assets that plot on the SML labeled $r_{z} \operatorname{LmBD}$. (See Grauer (1984) for a more detailed discussion of these and related issues.)

\section{Summary}

This paper has developed the efficient set mathematics for the case where MV problems are subject to general linear constraints. The efficient set mathematics follows from parametric quadratic programming problems, the optimality conditions for them, and the 


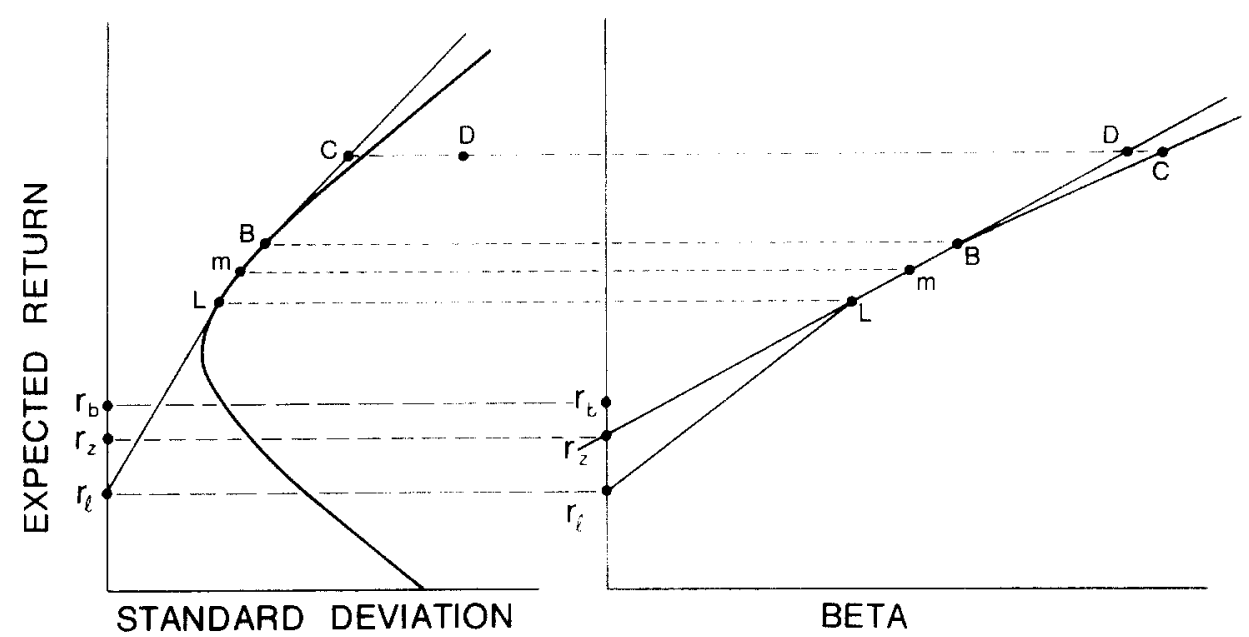

Figure 3. The MV CAPM with divergent borrowing and lending rates. The left-hand panel shows the MV efficient frontier, $r_{\ell} L m B C$. The market plots at $\mathrm{m}$. Portfolios containing risky securities only plot on the zero-beta SML, $r_{z} \mathrm{LmBD}$ in the right-hand panel. Feasible (infeasible) portfolios that either borrow or lend plot below (above) the SML; $r_{b}\left(r_{\ell}\right)$ itself plots above (below) the line by the distance $\lambda_{b} / t\left(\lambda_{l} / t\right)$. MV efficient portfolios that employ lending or borrowing plot on the line $r_{f} L$ or $B C$. According to the SML criterion these portfolios would be (incorrectly) classified as losers.

definitions of the expected return and variance of a portfolio. The framework allows us to generalize the traditional efficient set mathematics; all previous analyses represent special cases. We pointed out that the solutions and associated multipliers are piecewise linear functions of the PQP parameter $t$, or of the risk tolerance parameter $t$, which change in different intervals as the active set changes. Then we derived closed-form expressions for the optimal portfolio, the associated multipliers, and the portfolio's expected return and variance in each interval; identified where kinks in the efficient frontier occur; and showed that, in general, security means plot on a hyperplane spanned by the vector of betas and the gradients of the active constraints. In addition, we generalized the efficient set constants and showed that the multipliers have important roles in defining the zero-beta return, the deviations of securities from a line in mean-beta space, and in understanding kinks on the efficient frontier. Thus, at the individual investor's level, the analysis extends our knowledge of portfolio theory. At the market equilibrium level, it clarifies the zero-beta CAPM, the conditions under which securities plot on the SML, and provides insight into the ambiguities associated with using the SML to measure investment performance.

We thank the Natural Science and Engineering Research Council of Canada for financial support; Michael Brennan, Pao Cheng, Nils Hakansson, and John Heaney for valuable comments; and Ruth Cornale and Frederick Shen for most capable assistance. Naturally we are responsible for any errors.

\section{References}

Best, M. J. 1984. Equivalence of some quadratic programming algorithms. Mathematical Programming 30:71-87. 
Best, M. J. July 1982. An algorithm for the solution of the parametric quadratic programming problem. CORR Report 82-24. Waterloo, Ont.: University of Waterloo.

Best, M. J., and Grauer, R. R. September 1984. Sensitivity analysis for mean variance portfolio problems. Working paper. Burnaby, B.C.: Department of Economics, Simon Fraser University. (Revised December 1988).

Best, M. J., and Grauer, R. R. August 1985. The analytics of sensitivity analysis for mean variance portfolio problems. Working paper. Burnaby, B.C.: Department of Economics, Simon Fraser University.

Best, M. J., and Grauer, R. R. November 1986. Should investors hold well diversified portfolios? Working Paper. Burnaby, B.C.: Department of Economics, Simon Fraser University. (Revised November 1988).

Best, M. J., and Grauer, R. R. June 1987. Direct conditions for mean variance efficient portfolios to have positive weights: theory and evidence. Working Paper. Burnaby, B.C.: Department of Economics, Simon Fraser University. (Revised June 1989).

Best, M. J., and Ritter, K. 1985. Linear Programming: Active Set Analysis and Computer Programs. Englewood Cliffs, N.J.: Prentice-Hall.

Black, F. July 1972. Capital market equilibrium with restricted borrowing. Journal of Business 45:444-455.

Brennan, M. J. December 1971. Capital market equilibrium with divergent borrowing and lending rates. Journal of Financial and Quantitative Analysis 6:1197-1205.

Brennan, M. J. 1973. Taxes, market valuation and corporate financial policy. National Tax Journal 23:417-427.

Dybvig, P. H. March 1984. Short sales restrictions and kinks on the mean variance frontier. Journal of Finance 39:239-244.

Dybvig, P. H. March 1985. Acknowledgment: kinks on the mean-variance frontier. Journal of Finance 40:345.

Dybvig, P. H., and Ross, S. A. June 1985. The analytics of performance measurement using the security market line. Journal of Finance 40:401-416.

Grauer, R. R. September 1986. Normality, solvency and portfolio choice. Journal of Financial and Quantitative Analysis 21:265-278.

Grauer, R. R. April 1984. Further ambiguity when performance is measured by the security market line. Working paper. Burnaby, B.C.: Department of Economics, Simon Fraser University. (Revised February 1989).

Grauer, R. R., and Hakansson, N. April 1986. A half-century of returns on levered and unlevered portfolios of stocks, bonds, and bills, with and without small stocks. The Journal of Business 59:287-318.

Grauer, R. R., and Hakansson, N. July 1987. Gains from international diversification: 1968-85 returns on portfolios of stocks and bonds. Journal of Finance 42:721-739.

Grauer, R. R., and Hakansson, N. 1990. On timing the market: the empirical distribution approach with an inflation adapter. In progress.

Grauer, R. R., Hakansson, N. H., and Shen, F. C. 1990. Industry rotation in the U.S. stock market: 1934-1986 returns on passive, semi-passive, and active strategies. Journal of Banking and Finance. To appear.

Lintner, J. February 1965. The valuation of risky assets and the selection of risky investments in stock portfolios and capital budgets. Review of Economics and Statistics 47:13-47.

Litzenberger, R. H., and Ramaswamy, K. June 1979. The effect of personal taxes and dividends on capital asset prices: theory and empirical evidence. Journal of Financial Economics 7:163-195

Mangaserian, O. L. 1969. Nonlinear Programming. New York: McGraw-Hill.

Markowitz, H. March-June 1956. The optimization of a quadratic function subject to linear constraints. Naval Research Logistics Quarterly 111-133.

Markowitz, H. 1959. Portfolio selection: efficient diversification of investments. New York: John Wiley; New Haven: Yale University Press, 1970.

Mayers, D. 1972. Nonmarketable assets and the determination of capital market equilibrium under uncertainty. In Studies in the Theory of Capital Markets (Michael C. Jensen, ed.). New York: Praeger.

Merton, R. C. September 1972. An analytic derivation of the efficient portfolio frontier. Journal of Financial and Quantitative Analysis 7:1851-1872. 
Merton, R. C. December 1980. On estimating the expected return on the market: an exploratory investigation. Journal of Financial Economics 8:323-361.

Milne, F., and Smith, Jr., C. June 1980. Capital asset pricing with proportional transactions costs. Journal of Financial and Quantitative Analysis 15:253-266.

Ohlson, J. A. 1975. A general mean-variance approximation to expected utility as the trading interval approaches zero. In Stochastic Optimization Models in Finance (W. T. Ziemba and R. G. Vickson, eds.). New York: Academic Press.

Pulley, L. B. September 1981. A general mean-variance approximation to expected utility for short holding periods. Journal of Financial and Quantitative Analysis 16:361-373.

Roll, R. March 1977. A critique of the asset pricing theory's tests; part 1: on past and potential testability of the theory. Journal of Financial Economics 4:129-176.

Roll, R. December 1980. Orthogonal portfolios. Journal of Financial and Quantitative Analysis 15:1005-1023.

Ross, S. A. March 1977. The capital asset pricing model (CAPM), short-sale restrictions and related issues. Journal of Finance 32:177-183.

Sharpe, W. F. September 1964. Capital asset prices: a theory of market equilibrium under conditions of risk. Journal of Finance 19:425-442.

Sharpe, W. F. 1970, Portfolio Theory and Capital Markets. New York: McGraw-Hill.

Sharpe, W. F. 1985. Investments (3rd ed.). Englewood Cliffs, N.J.: Prentice-Hall.

Stulz, R. M. September 1981. On the effects of barriers to international investment. Journal of Finance 36:923-934. 